\title{
ON THE LENGTH OF ASYMPTOTIC PATHS OF MEROMORPHIC FUNCTIONS OF ORDER ZERO
}

\section{SAKARI TOPPILA}

\section{Introduction}

We use the usual notation of the Nevanlinna theory. We shall consider the following problem of Erdös (Clunie and Hayman [2, Problem 2.41]): Suppose that $f$ is an entire function of finite order and $\Gamma$ is a locally rectifiable path on which $f(z) \rightarrow \infty$. Let $l(r, \Gamma)$ be the length of $\Gamma$ in $|z| \leqq r$. Find a path for which $l(r, \Gamma)$ grows as slowly as possible and estimate $l(r, \Gamma)$ in terms of $M(r, f)$. If $f$ has zero order, or, more generally, finite order, can a path $\Gamma$ be found for which $l(r, \Gamma)=O(r)$ as $r \rightarrow \infty$ ?

First we shall consider the case when we may choose a ray for $\Gamma$. We denote by $U(a, r)$ the open disc $|z-a|<r$. Following Hayman [4], we say that the union of the open discs $U\left(a_{n}, r_{n}\right), n=1,2, \ldots$, is an $\varepsilon$-set if $a_{n} \rightarrow \infty$ as $n \rightarrow \infty$ and the series $\sum r_{n} /\left|a_{n}\right|$ converges. We note that if $f(z) \rightarrow a$ as $z \rightarrow \infty$ outside an $\varepsilon$-set, then $a$ is a radial asymptotic value of $f$; in fact, $f\left(r e^{i \theta}\right) \rightarrow a$ as $r \rightarrow \infty$ for almost all $\theta$. Hayman [4] has shown that if $f$ is an entire function satisfying $T(r, f)=O\left((\log r)^{2}\right)$, then

$$
\log |f(z)|=(1+o(1)) T(|z|, f)
$$

as $z \rightarrow \infty$ outside an $\varepsilon$-set. Anderson [1] proved that if $f$ is a meromorphic function such that $\delta(\infty, f)>0$ and

then

$$
T(2 r, f)-T(r, f)=o(T(r, f))(\log \log r)^{-1},
$$

$$
\lim \inf \frac{\log |f(z)|}{T(|z|, f)} \geqq \delta(\infty, f)
$$

as $z \rightarrow \infty$ outside an $\varepsilon$-set. We shall prove the following theorem.

Theorem 1. Suppose that $f$ is meromorphic in the finite complex plane $C$ and that $\delta(\infty, f)=\delta>0$. If there exists $m, 0<m<\log 2$, such that

$$
T(2 r, f) \leqq T(r, f)\left(1+\frac{m \delta}{\log \log r}\right)
$$

for all large values of $r$, then $f(z) \rightarrow \infty$ as $z \rightarrow \infty$ outside an $\varepsilon$-set. 
The growth condition (1) with $m<\log 2$ is more or less the best possible. The question whether $m$ can be replaced by $\log 2$ in (1) remains open, but we shall show that $m$ cannot be larger than $\log 2$.

Theorem 2. For any $\delta$ and $m, 0<\delta \leqq 1, m>\log 2$, there exists a transcendental meromorphic function $f$ ( $f$ entire if $\delta=1$ ) such that $\delta(\infty, f)=\delta$, f has no radial asymptotic values, and

$$
T(2 r, f) \leqq T(r, f)\left(1+\frac{m \delta}{\log \log r}\right)
$$

for all large values of $r$.

On the other hand, Goldberg and Eremenko [3] and the author [7] have proved that if $\varphi(r) \rightarrow \infty$ as $r \rightarrow \infty$, then there exists an entire function $f$ satisfying

$$
T(r, f)=O\left(\varphi(r)(\log r)^{2}\right)
$$

such that if $\Gamma$ is any asymptotic path of $f$, then $l(r, \Gamma) \neq O(r)$. There arises the question whether an entire function $f$ satisfying (3) can be constructed such that

$$
\liminf _{r \rightarrow \infty} \frac{l(r, \Gamma)}{r}>1
$$

for any asymptotic path $\Gamma$. We shall give a negative answer to this question.

Theorem 3. If $f$ is an entire function satisfying

$$
T(r, f)=O\left((\log r)^{M}\right)
$$

for some $M>0$, then there exists an asymptotic path $\Gamma$ such that

$$
\liminf _{r \rightarrow \infty} \frac{l(r, \Gamma)}{r}=1
$$

Furthermore, we prove the following two theorems.

Theorem 4. Let $f$ be an entire function of order zero. Then there exists an asymptotic path $\Gamma$ such that

$$
l(r, \Gamma)=o\left(r^{1+\varepsilon}\right)
$$

for any $\varepsilon>0$.

Theorem 5. If $f$ is an entire function of order zero satisfying

$$
\liminf _{r \rightarrow \infty} \frac{n\left(r^{1+\varepsilon}, 0, f\right)}{T(r, f)}=0
$$

for some $\varepsilon>0$, then there exists an asymptotic path $\Gamma$ satisfying (5). 


\section{Proof of Theorem 1}

Let $f$ satisfy the hypotheses of Theorem 1. Following Anderson [1], we choose a finite $b$ such that

$$
N(r, b)=T(r, f)+O\left((T(r, f))^{3 / 4}\right) .
$$

Then it follows from (1) that

$$
\begin{aligned}
n(r, b) \log 2 & \leqq N(2 r, b)-N(r, b) \\
& \leqq T(2 r, f)-T(r, f)+O\left((T(r, f))^{3 / 4}\right) \\
& \leqq(1+o(1)) m \delta T(r, f)(\log \log r)^{-1} .
\end{aligned}
$$

Let $k$ be a positive integer. We choose $\varrho$ such that

$$
\log \left(\frac{\varrho}{2^{k+3}}\right)=-\lambda \log \log 2^{k}
$$

where $\lambda=((\log 2) / m)^{1 / 2}>1$. From the proof of Theorem 2 of Anderson [1] it follows that for $2^{k} \leqq|z|<2^{k+1}$

$$
\log |f(z)-b| \geqq(\delta+o(1)) T(|z|, f)+n\left(2^{k+2}, b\right) \log \left(\varrho / 2^{k+3}\right)
$$

outside a set of circles in $2^{k-1} \leqq|z| \leqq 2^{k+2}$, the sum of whose radii is at most $32 \varrho$. Let $z, 2^{k} \leqq|z|<2^{k+1}$, lie outside these discs. From (i) and (ii) it follows that

$$
n\left(2^{k+2}, b\right) \log \left(\varrho / 2^{k+3}\right) \geqq-(1+o(1)) \lambda^{-1} \delta T\left(2^{k+2}, f\right),
$$

and since $T(8 r, f)=(1+o(1)) T(r, f)$, we conclude from (iii) that

$$
\log |f(z)|>\left(\delta\left(1-\lambda^{-1}\right)+o(1)\right) T(|z|, f)>(1+o(1)) \log |z| .
$$

Thus $\log |f(z)| \geqq(1+o(1)) \log |z|$ outside a set of circles the sum of whose radii taken over circles meeting the set $2^{k} \leqq|z| \leqq 2^{k+1}$ is at most

$$
O\left(2^{k} \exp \left(-\lambda \log \log 2^{k}\right)\right)=O\left(2^{k} k^{-\lambda}\right)
$$

These circles subtend angles at the origin whose sum is $O\left(k^{-\lambda}\right)$. Since $\lambda>1$, the series $\sum k^{-\lambda}$ converges, and we conclude that $f(z) \rightarrow \infty$ as $z \rightarrow \infty$ outside an $\varepsilon$-set. This proves Theorem 1.

\section{Proof of Theorem 2}

Let $m>\log 2$ and $\delta, 0<\delta \leqq 1$, be given. Let $M>10$ be a positive integer such that

$$
m\left(1-e^{-M}\right)\left(1-2^{-M}\right)>\log 2 .
$$

We denote

(ii)

$$
\alpha=2^{M} \delta^{-1}\left(1-e^{-M}\right)\left(1-2^{-M}\right)^{2},
$$


and for $k=1,2, \ldots$, we set $r_{k}=k^{\alpha k}$ and $a_{k}=r_{k} e^{i \varphi_{k}}$, where the angles $\varphi_{k}$ will be specified later. Let $t_{1}=2^{M}$ and $t_{k}=2^{M k}-2^{M(k-1)}$ for $k \geqq 2$.

We shall consider the function

$$
f_{1}(z)=\prod_{k=1}^{\infty}\left(1-\frac{z}{a_{k}}\right)^{t_{k}}
$$

The sequence $t_{k}$ is chosen such that $n\left(r, 0, f_{1}\right)=2^{M k}$ for $r_{k} \leqq r<r_{k+1}$, and we see from the choice of $r_{k}$ that

$$
N\left(r_{k+1}, 0, f_{1}\right)-N\left(r_{k}, 0, f_{1}\right)=2^{M k} \log \left(r_{k+1} / r_{k}\right)=(1+o(1)) 2^{M k} \alpha \log k .
$$

This implies that

$$
\begin{gathered}
N\left(r_{k+1}, 0, f_{1}\right)=(1+o(1)) 2^{M k} \alpha \log k\left(1+2^{-M}+2^{-2 M}+\ldots\right) \\
=(1+o(1)) \alpha 2^{M k}\left(1-2^{-M}\right)^{-1} \log k .
\end{gathered}
$$

Now we see that $n\left(r, 0, f_{1}\right)=o\left(N\left(r, 0, f_{1}\right)\right)$ and therefore $N\left(2 r, 0, f_{1}\right)=(1+o(1))$.

- $N\left(r, 0, f_{1}\right)$. Using Lemma 1 of Anderson [1] we conclude that

$$
\log M\left(r, f_{1}\right)=(1+o(1)) N\left(r, 0, f_{1}\right)=(1+o(1)) T\left(r, f_{1}\right) .
$$

Then $f$ satisfies the condition

$$
\log M\left(2 r, f_{1}\right)=(1+o(1)) \log M\left(r, f_{1}\right),
$$

and it follows from Theorem 2 of Anderson [1] that

$$
\log \left|f_{1}(z)\right|=(1+o(1)) \log M\left(r, f_{1}\right)=(1+o(1)) N\left(r, 0, f_{1}\right)
$$

$(r=|z|)$ outside the union of the $\operatorname{discs} U\left(a_{n}, r_{n} / 4\right)$.

If $\delta=1$, we set $f_{2}(z) \equiv 1$, and if $0<\delta<1$, then

$$
f_{2}(z)=\prod_{k=1}^{\infty}\left(1+\frac{z}{a_{k}}\right)^{s_{k}},
$$

where the sequence $s_{k}$ of positive integers is chosen such that

for any $k$. Then

$$
\left|n\left(r_{k}, 0, f_{2}\right)-(1-\delta) 2^{M k}\right| \leqq \frac{1}{2}
$$

$$
\left|n\left(r, 0, f_{2}\right)-(1-\delta) n\left(r, 0, f_{1}\right)\right| \leqq \frac{1}{2}
$$

and, as above, we see that

$$
\log \left|f_{2}(z)\right|=(1+o(1))(1-\delta) N\left(|z|, 0, f_{1}\right)
$$

outside the union of the discs $U\left(-a_{n}, r_{n} / 4\right)$.

We set $f(z)=f_{1}(z) / f_{2}(z)$. Then $f$ is entire if $\delta=1$, and for any $\delta, 0<\delta \leqq 1, f$ satisfies

$$
\log |f(z)| \geqq(1+o(1)) \delta N(|z|, 0, f)
$$


outside the union of the discs $U\left(a_{n}, r_{n} / 4\right)$, and on the boundary of these discs

$$
\log |f(z)|=(1+o(1)) \delta N(|z|, 0, f) .
$$

We choose a finite $b$ such that

$$
N(r, b, f)=T(r, f)+O\left((T(r, f))^{3 / 4}\right) .
$$

Using Rouche's theorem, we see from (iv) that for all large values of $k$,

$$
n(r, b, f)=n(r, 0, f)=2^{M(k-1)}
$$

if $(5 / 4) r_{k-1} \leqq r \leqq(3 / 4) r_{k}$, and

$$
2^{M(k-1)} \leqq n(r, b, f) \leqq 2^{M k}
$$

if $(3 / 4) r_{k} \leqq r \leqq(3 / 4) r_{k+1}$. Therefore we see from (iii) that if $(5 / 4) r_{k-1} \leqq r<(5 / 4) r_{k}$, then

$$
\begin{aligned}
& N(2 r, b, f)-N(r, b, f) \leqq n(2 r, b, f) \log 2 \\
& \leqq(1+o(1)) N(r, 0, f) \frac{2^{M}\left(1-2^{-M}\right) \log 2}{\alpha \log k} .
\end{aligned}
$$

This implies together with (vi) and (ii) that

$$
T(2 r, f)-T(r, f) \leqq(1+o(1)) T(r, f) \frac{\delta \log 2}{\left(1-e^{-M}\right)\left(1-2^{-M}\right) \log k},
$$

and we see from (i) that $f$ satisfies the condition (2) for all large values of $r$.

Comparing the growth of $N(r, b, f)$ and $N(r, 0, f)$ we see easily that

$$
N(r, b, f)=(1+o(1)) N\left(r, 0, f^{\prime}\right) .
$$

Then we have $N(r, 0, f)=(1+o(1)) T(r, f)$, and since

$$
N(r, \infty, f)=N\left(r, 0, f_{2}\right)=(1+o(1)) N(r, 0, f)(1-\delta),
$$

we conclude that $\delta(\infty, f)=\delta$.

The function $h_{n}(z)=f(z)\left(1-z / a_{n}\right)^{-t_{n}}$ is analytic in $\left|z-a_{n}\right| \leqq r_{n} / 4$, and we see from (v) that

$$
\log \left|h_{n}(z)\right| \leqq(1+o(1)) \delta N\left(r_{n}, 0, f\right)+t_{n} \log 4 \leqq(1+o(1)) \delta N\left(r_{n}, 0, f\right)
$$

on the boundary of $U\left(a_{n}, r_{n} / 4\right)$. Then it follows from the maximum principle that $h_{n}$ satisfies (vii) in $U\left(a_{n}, r_{n} / 4\right)$. We define $\varrho_{n}$ by the equation

$$
\log \left(r_{n} / \varrho_{n}\right)=\left(1-e^{-2 M}\right) \log n .
$$

We see from (vii) that if $z$ lies on the boundary of $U\left(a_{n}, \varrho_{n}\right)$, then

$$
\begin{aligned}
& \log |f(z)|=\log \left|h_{n}(z)\right|-t_{n} \log \left(r_{n} / \varrho_{n}\right) \\
& \leqq(1+o(1)) \delta N\left(r_{n}, 0, f\right)-t_{n} \log \left(r_{n} / \varrho_{n}\right) .
\end{aligned}
$$

This implies together with (iii) and (viii) that

$$
\log |f(z)| \leqq-(1+o(1))\left(e^{-M}-e^{-2 M}\right) 2^{M n}\left(1-2^{-M}\right) \log n \leqq-(1+o(1)) \log n
$$


in $U\left(a_{n}, \varrho_{n}\right)$, and we see that if $z$ tends to infinity through the union of the discs $U\left(a_{n}, \varrho_{n}\right)$, then $f(z)$ tends to zero.

We assume now that the angles $\varphi_{k}$ are chosen such that $\varphi_{1}=0$ and $\varphi_{k+1}=$ $\varphi_{k}+\varrho_{k} / r_{k}$ for $k \geqq 1$. If $\varphi_{k} \leqq \varphi \leqq \varphi_{k+1}$ and $\left|z_{0}\right|<\varrho_{k} / 8$, then the ray $z=z_{0}+r e^{i \varphi}$ meets at most one of the discs $U\left(a_{k}, \varrho_{k}\right)$ and $U\left(a_{k+1}, \varrho_{k+1}\right)$. It follows from (viii) that $\varrho_{n} \rightarrow \infty$ as $n \rightarrow \infty$ and that the series $\sum \varrho_{n} / r_{n}$ diverges. Therefore any ray $z=$ $z_{0}+r e^{i \varphi}$ meets infinitely many of the discs $U\left(a_{n}, \varrho_{n}\right)$ and so

$$
\liminf _{r \rightarrow \infty}\left|f\left(z_{0}+r e^{i \varphi}\right)\right|=0
$$

for any fixed complex $z_{0}$ and real $\varphi$. On the other hand, it follows from (iv) that

$$
\limsup _{r \rightarrow \infty}\left|f\left(z_{0}+r e^{i \varphi}\right)\right|=\infty
$$

for any fixed $z_{0}$ and $\varphi$, and we conclude that $f$ has no radial asymptotic values. This completes the proof of Theorem 2 .

\section{Proof of Theorem 4}

Let $f$ be an entire function of order zero. We may suppose that $f$ has no radial asymptotic values because otherwise we could choose a ray for the desired path $\Gamma$. We choose a continuous path $\gamma:[0,1) \rightarrow C$ such that $\gamma(0)=0, \gamma(t) \rightarrow \infty$ as $t \rightarrow 1$ and

(i)

$$
\log |f(z)| \geqq 3 \log |z|
$$

on $\gamma$ for all large values of $|z|$. We denote

$$
B=\{z \in C: \log |f(z)| \leqq \log |z|\} .
$$

Using (i), we choose $t_{0}, 0<t_{0}<1$, such that

$$
\log |f(\gamma(t))| \geqq 3 \log |\gamma(t)|>9
$$

for $t \geqq t_{0}$. We choose $\varrho_{0}>0$ such that $U\left(\gamma\left(t_{0}\right), \varrho_{0}\right)$ is contained in the complement of $B$ and that the circle $\left|z-\gamma\left(t_{0}\right)\right|=\varrho_{0}$ contains at least one point of $B$. Inductively, if $t_{k-1}$ and $\varrho_{k-1}(k \geqq 1)$ are determined, we choose $t_{k}$ to be the greatest value of $t$ such that the open disc

$$
U\left(\gamma\left(t_{k}\right),\left|\gamma\left(t_{k}\right)-\gamma\left(t_{k-1}\right)\right|-\varrho_{k-1}\right)
$$

does not contain any point of $B$ and that the boundary of this disc contains at least one point of $B$. The radius of this disc is denoted by $\varrho_{n}$ and, for the sake of simplicity, we write $C_{k}=U\left(\gamma\left(t_{k}\right), \varrho_{k}\right)$. Since $\infty$ is not a radial asymptotic value of $f$, we see that our process gives a denumerable collection of discs $C_{k}$. From the continuity of $f$ we conclude that the points $\gamma\left(t_{k}\right)$ cannot have any finite point $z$ as a limit point. Then $\gamma\left(t_{k}\right) \rightarrow \infty$ as $k \rightarrow \infty$ and, using again the fact that $\infty$ is not a radial asymptotic value 
of $f$, we note that

(iii)

$$
\lim _{k \rightarrow \infty}\left(\left|\gamma\left(t_{k}\right)\right|-\varrho_{k}\right)=\infty \text {. }
$$

The open discs $C_{k}$ are mutually disjoint and the boundary circles of $C_{k}$ and $C_{k+1}$ have exactly one point in common. Since all the discs $C_{k}$ are contained in the complement of $B$, we deduce now that $\log |f(z)| \geqq \log |z|$ on that segment which joins the points $\gamma\left(t_{k}\right)$ and $\gamma\left(t_{k+1}\right)$. Let $\Gamma$ be the path consisting of these segments. It follows from (iii) that $\Gamma$ is a path going from $\gamma\left(t_{0}\right)$ to $\infty$, and since $\log |f(z)| \geqq \log |z|$ on $\Gamma$, we deduce that $f(z) \rightarrow \infty$, as $z \rightarrow \infty$ along $\Gamma$.

We denote by $a_{n}, n=1,2, \ldots$, the zeros of $f$, and for any finite $z$ we set $\omega(z)=$ $\min \left\{\left|z-a_{n}\right|: n=1,2, \ldots\right\}$. Let $r>4$ and $|z|<4 r$. Then the logarithmic derivative of $f$ satisfies

$$
\left|\frac{f^{\prime}(z)}{f(z)}\right|=\left|\sum_{n=1}^{\infty} \frac{1}{z-a_{n}}\right| \leqq \omega(z)^{-1} n\left(r^{4}, 0\right)+2 \sum_{\left|a_{n}\right|>r^{4}}\left|a_{n}\right|^{-1} .
$$

Since $f$ is of order zero,

$$
\sum_{\left|a_{n}\right|>r^{4}}^{\infty}\left|a_{n}\right|^{-1} \leqq \sum_{k=4}^{\infty} r^{-k} n\left(r^{k+1}, 0\right)=o\left(r^{-2}\right),
$$

and we deduce that

$$
\left|\frac{f^{\prime}(z)}{f(z)}\right| \leqq \omega(z)^{-1} n\left(r^{4}, 0\right)+o\left(r^{-2}\right)
$$

in $|z| \leqq 4 r$.

We denote by $l(A)$ the length measure of $A$ if $A$ is a set consisting of a finite number of rectifiable arcs. For $k \geqq 1$, the set $\Gamma \cap C_{k}$ consists of two radii of $C_{k}$. These radii are denoted by $\alpha_{k}$ and $\beta_{k}$; then clearly $l\left(\alpha_{k}\right)=l\left(\beta_{k}\right)=\varrho_{k}$ and $l\left(\Gamma \cap C_{k}\right)=$ $2 \varrho_{k}$. We denote

$$
\Gamma_{r}=\Gamma \cap\{z: r \leqq|z| \leqq 2 r\} .
$$

If $\Gamma_{r} \cap \beta_{k} \neq \emptyset$ and $\varrho_{k} \geqq r / 4$, we choose an open disc $D_{k}$ with radius $d_{k} \geqq r / 8$ such that

$$
\Gamma_{r} \cap \beta_{k} \subset D_{k} \subset C_{k} \cap U(0,3 r) .
$$

Then $l\left(\Gamma_{r} \cap \beta_{k}\right) \leqq 2 d_{k}$ and the area $\pi d_{k}^{2}$ of $D_{k}$ satisfies the inequality

$$
16 \pi d_{k}^{2} \geqq \pi r l\left(\Gamma_{r} \cap \beta_{k}\right) .
$$

The discs $D_{k}$ are mutually disjoint and all of them are contained in $U(0,3 r)$. Therefore the sum of the areas of $D_{k}$ is at most $9 \pi r^{2}$, and we deduce from (v) that

$$
\sum_{\beta_{k} \geqq r / 4} l\left(\Gamma_{r} \cap \beta_{k}\right) \leqq 144 r=O(r) .
$$

In the same manner, we get the estimate

and conclude that

$$
\sum_{\alpha_{k} \geqq r / 4} l\left(\Gamma_{r} \cap \alpha_{k}\right)=O(r)
$$

$$
l\left(\Gamma_{r} \cap \bigcup_{\varrho_{k} \geqq r / 4} C_{k}\right)=O(r) .
$$


Let $C_{p}$ contain at least one point of $r \leqq|z| \leqq 2 r$ and let the radius $\varrho_{p}$ satisfy

$$
r / 2^{k+2} \leqq \varrho_{p}<r / 2^{k+1}
$$

for some positive integer $k$. Then we have $l\left(\Gamma_{r} \cap C_{p}\right) \leqq l\left(\Gamma \cap C_{p}\right)=2 \varrho_{p}<r / 2^{k}$ and

$$
\varrho_{p}^{2} \geqq l\left(\Gamma_{r} \cap C_{p}\right) r / 2^{k+3} .
$$

On the boundary of $C_{p}$ there exists a point $b$ such that $\log |f(b)|=\log |b|$. Let $J$ be the segment joining $b$ and the centre $\gamma\left(t_{p}\right)$ of $C_{p}$. Then it follows from (ii) and (iv) that

$$
\begin{aligned}
\log r & \leqq 3 \log \left|\gamma\left(t_{p}\right)\right|-\log |b| \leqq \log \left|f\left(\gamma\left(t_{p}\right)\right)\right|-\log |f(b)| \\
\leqq & \int_{J} \frac{f^{\prime}(z)}{f(z)} d z \mid \leqq \varrho_{p}\left(\frac{n\left(r^{4}, 0\right)}{\omega\left(\gamma\left(t_{p}\right)\right)-\varrho_{p}}+o\left(r^{-2}\right)\right) .
\end{aligned}
$$

For large values of $r$ this implies that $\omega\left(\gamma\left(t_{p}\right)\right) \leqq(1 / 8) \varrho_{p} n\left(r^{4}, 0\right)$, and we conclude from (vii) that there exists $n, 1 \leqq n \leqq n\left(r^{4}, 0\right)$, such that

$$
C_{p} \subset U\left(a_{n}, n\left(r^{4}, 0\right) r / 2^{k+1}\right) .
$$

Since the discs $C_{p}$ are mutually disjoint, we see by comparing the areas from (viii) that

$$
\frac{r}{2^{k+3}} \sum l\left(\Gamma_{r} \cap C_{p}\right) \leqq n\left(r^{4}, 0\right)\left(\frac{n\left(r^{4}, 0\right) r}{2^{k+1}}\right)^{2},
$$

where the sum is taken over those $p$ which satisfy (vii). This implies that

$$
l\left(\Gamma_{r} \cap \bigcup_{\varrho_{p}<1 / 4} C_{p}\right) \leqq 4 r\left(n\left(r^{4}, 0\right)\right)^{3} \sum_{k=1}^{\infty} 2^{-k-1}
$$

for all large values of $r$.

Let $\varepsilon>0$ be given. We choose $\alpha$ such that $1<\alpha<1+\varepsilon$. Since $f$ is of order zero, we see from (vi) and (ix) that $l\left(\Gamma_{r}\right)=o\left(r^{\alpha}\right)$. We choose $r_{0}$ such that $l\left(\Gamma_{r}\right)<r^{\alpha}$ for $r \geqq r_{0}$. We get for $r>r_{0}$

$$
l(r, \Gamma) \leqq l\left(2 r_{0}, \Gamma\right)+\sum_{k=0}^{\infty}\left(\frac{r}{2^{k}}\right)^{\alpha} \leqq l\left(2 r_{0}, \Gamma\right)+2 r^{\alpha},
$$

and therefore we have $l(r, \Gamma)=o\left(r^{1+\varepsilon}\right)$. This completes the proof of Theorem 4 .

\section{Proof of Theorems 5 and 3}

Let $f$ be an entire function of order zero, $\varepsilon>0$, and let there exist a sequence $r_{n}$ such that $\lim r_{n}=\infty$ and $n\left(r_{n}^{1+\varepsilon}, 0\right)=o\left(T\left(r_{n}, f\right)\right)$. We choose $\alpha$ by the equality $\alpha^{5}=1+\varepsilon$. The method used by Anderson [1] in the proofs of Theorems 1 and 2 is directly applicable in the ring domains $r_{n}^{\alpha} \leqq|z| \leqq r_{n}^{\alpha^{4}}$, and we may conclude that there exist circles $C_{n}:|z|=\varrho_{n}, r_{n}^{\alpha} \leqq \varrho_{n} \leqq 2 r_{n}^{\alpha}, C_{n}^{\prime}:|z|=R_{n}, r_{n}^{\alpha^{3}} \leqq R_{n} \leqq 2 r_{n}^{\alpha^{3}}$, and a path $\gamma_{n}$ joining the circles $C_{n}$ and $C_{n}^{\prime}$ with $l\left(\gamma_{n}\right)=(1+o(1)) R_{n}$ such that $\log |f(z)|>$ $(1 / 2+o(1)) T(|z|, f)$ on $C_{n}^{\prime} \cup C_{n} \cup \gamma_{n}$. From Theorem 4 it follows that there exists 
an asymptotic path $\Gamma_{0}$ such that $l\left(r, \Gamma_{0}\right)=o\left(r^{\alpha}\right)$. Using $C_{n}, C_{n}^{\prime}$ and $\gamma_{n}$ we may modify $\Gamma_{0}$ into a new asymptotic path $\Gamma$ such that

$$
l\left(R_{n}-1, \Gamma\right) \leqq l\left(\Gamma_{0}, \varrho_{n}\right)+2 \pi \varrho_{n}+l\left(\gamma_{n}\right)=o\left(\left(2 r_{n}^{\alpha}\right)^{\alpha}\right)+O\left(r_{n}^{\alpha}\right)+(1+o(1)) R_{n} .
$$

Since $R_{n} \geqq r_{n}^{\alpha^{3}}$, we conclude now that $l\left(R_{n}-1, \Gamma\right)=(1+o(1))\left(R_{n}-1\right)$. This proves Theorem 5.

Let us suppose that $f$ is an entire function satisfying (4). We choose $\delta>0$ such that $T(r, f)=O\left((\log r)^{\delta+1 / 2}\right)$ and $T(r, f) \neq O\left((\log r)^{\delta}\right)$. Then $n(r, 0, f)=$ $O\left((\log r)^{\delta-1 / 2}\right)$, and there exist arbitrarily large values of $r$ such that $T(r, f)>$ $(\log r)^{\delta}$. For these values of $r$ we have

$$
\frac{n\left(r^{2}, 0, f\right)}{T(r, f)} \leqq \frac{O\left((\log r)^{\delta-1 / 2}\right)}{(\log r)^{\delta}}=O\left((\log r)^{-1 / 2}\right)=o(1)
$$

and Theorem 3 follows from Theorem 5 .

Remark. I thank Doctor J. M. Anderson for informing me that my Theorem 4 is essentially contained in Theorem 1 of Chang Kuan-Heo [6].

\section{References}

[1] Anderson, J. M.: Asymptotic values of meromorphic functions of smooth growth. - Glasgow Math. J. 20, 1979, 155-162.

[2] Clunie, J., and W. K. Hayman: Proceedings of the symposium on complex analysis, Canterbury, 1973. - London Mathematical Society Lecture Note Series 12, Cambridge University Press, Cambridge, 1974.

[3] Gol'dberg, A. A., and A. È. Eremenko: On asymptotic curves of entire functions of finite order. - Math. USSR-Sb. 37: 4, 1980, 509-533.

[4] HaYman, W. K.: Slowly growing integral and subharmonic functions. - Comment. Math. Helv. 34, 1960, 75-84.

[5] Hayman, W. K.: On Iversen's theorem for meromorphic functions with few poles. - Acta Math. 141, 1978, 115-145.

[6] Kuan-Heo, Chang: Asymptotic values of entire and meromorphic functions. - Sci. Sinica 20: 6, $1977,720-739$.

[7] Toppila, S.: On the length of asymptotic paths of entire functions of order zero. - Ann. Acad. Sci. Fenn. Ser. A. I. Math. 5, 1980, 13-15.

University of Helsinki

Department of Mathematics

SF-00100 Helsinki 10

Finland

Received 28 September 1983 